\title{
Menadione Sodium Bisulfite-Protected Tomato Leaves against Grey Mould via Antifungal Activity and Enhanced Plant Immunity
}

\author{
Yeon Sook Jo ${ }^{1}$, Hye Bin Park ${ }^{1}$, Ji Yun Kim ${ }^{1}$, Seong Min Choi ${ }^{1}$, Da Sol Lee ${ }^{1}$, Do Hoon Kim¹, Young Hee Lee', \\ Chang-Jin Park ${ }^{2}$, Yong-Chull Jeun ${ }^{3}$, and Jeum Kyu Hong (iD) ${ }^{1 *}$ \\ ${ }^{I}$ Department of Horticultural Science, Gyeongnam National University of Science and Technology (GNTech), Jinju 52725, \\ Korea \\ ${ }^{2}$ Department of Bioresources Engineering, Sejong University, Seoul 05006, Korea \\ ${ }^{3}$ College of Applied Life Science, Faculty of Bioscience and Industry, The Research Institute for Subtropical Agriculture \\ and Biotechnology, Jeju National University, Jeju 63243, Korea
}

https://doi.org/10.5423/PPJ.OA.06.2020.0113

Plant Pathol. J. 36(4) : 335-345 (2020)

In this paper, the name of the first author was incorrectly spelled. The correct spelling of the author's name is Yeon Sook Jo. The corrected version of author list is as follows. The authors would like to apologize for any inconvenience caused.

\section{Original version}

Youn Sook Jo ${ }^{1}$, Hye Bin Park ${ }^{1}$, Ji Yun Kim ${ }^{1}$, Seong Min Choi ${ }^{1}$, Da Sol Lee ${ }^{1}$, Do Hoon Kim ${ }^{1}$, Young Hee Lee ${ }^{1}$, Chang-Jin Park $^{2}$, Yong-Chull Jeun ${ }^{3}$, and Jeum Kyu Hong ${ }^{1 *}$

\section{Corrected version}

Yeon Sook Jo ${ }^{1}$, Hye Bin Park ${ }^{1}$, Ji Yun Kim ${ }^{1}$, Seong Min Choi ${ }^{1}$, Da Sol Lee ${ }^{1}$, Do Hoon Kim ${ }^{1}$, Young Hee Lee ${ }^{1}$, Chang-Jin Park $^{2}$, Yong-Chull Jeun ${ }^{3}$, and Jeum Kyu Hong ${ }^{1 *}$

\footnotetext{
*Corresponding author.

Phone) +82-55-751-3251, FAX) +82-55-751-3257

E-mail) jkhong@gntech.ac.kr

ORCID

Jeum Kyu Hong

https://orcid.org/0000-0002-9161-511X

(c) This is an Open Access article distributed under the terms of the Creative Commons Attribution Non-Commercial License (http://creativecommons.org/licenses/by-nc/4.0) which permits unrestricted noncommercial use, distribution, and reproduction in any medium, provided the original work is properly cited.
}

Articles can be freely viewed online at www.ppjonline.org. 